\title{
Schmerz: Psychosomatisches Grundphänomen, Krankheitsfaktor und Leiden
}

$\mathrm{D}$ ie Bedeutung des Schmerzes erschöpft sich nicht in der Funktion als Warnsignal im biologischen Leben und Überleben, als Epiphänomen von Schädigungen und Erkrankungen. Es handelt sich vielmehr um das erste Principium individuationis, das im menschlichen Leben und in der Ich-Entwicklung zum Erkennen von Identität und Verschiedenheit führt, und hypothetisch um das Verbindungsglied zwischen Soma und Psyche. Schmerz ist Korrektiv menschlicher Reifung und psychosozialer Entwicklung.

Schon bei akutem Auftreten ist Schmerz auch krankmachende Kraft mit Auswirkungen auf Herz-Kreislauffunktion und Ventilation, Magen-Darm-Trakt und Urogenitalsystem, die Schlaflosigkeit, Angst und Immobilität bewirken kann. Bei rezidivierenden und vor allem bei chronischen, d.h. lang anhaltenden, täglich auftretenden Schmerzsyndromen treten die nützlichen, lebenserhaltenden Gesichtspunkte in den Hintergrund. Sie können Berufsleben und soziale Aktivitäten stark beieinträchtigen, die Freizeit vergällen und die Lebensqualität ganz erheblich verschlechtern.

Deshalb ist es verdienstvoll, wenn Hans-Christoph Diener wiederkehrend den aktuellen Wissensstand bei Migräne umreißt. In der Natur des empirischen Vorgehens liegt es, dass immer nur vorläufige, keine endgültigen Behandlungsergebnisse vorgelegt werden können. Im Migräneanfall übertrifft die Wirkung einfacher Analgetika wie Acetylsalicylsäure und Paracetamol oder der nicht-steroidalen Antirheumatika Ibuprofen, Diclofenac und Naproxen mit einer Erfolgsquote von 30$40 \%$ diejenige von Plazebo nur geringfügig. Etwa doppelt so hoch ist hingegen die Ansprechrate auf spezifisch wirkende Triptane. Für manche Migränepatienten ist es ein grundlegender Nutzen, wenn sie dadurch Angst und Ausgeliefertsein überwinden und ihre Lebensführung ganz anders gestalten können. Aber auch hier wird mit Hilfe der verschiedenen Substanzen jeweils nur ein Teil der Betroffenen erreicht, so dass die Wirkung immer evaluiert und möglichst auch dokumentiert werden muss. Dieners Folgerung, dass bei häufiger Migräne medikamentöse und nicht-medikamentöse Therapien kombiniert werden sollten, ist uneingeschränkt zuzustimmen. Bezüglich der empfohlenen Verfahren Stressbewältigung, Ausdauersport und Akupunktur besteht allerdings weiterer Erklärungsbedarf mit empirischwissenschaftlichen Belegen.
Aus der Vielfalt verschiedener Schmerzsyndrome bei Parkinsonpatienten, die Arda Yazici umreißt, geht die Komplexität der Problematik mit der Erfordernis eingehender Schmerzanalyse, sorgfältiger Untersuchung und interdisziplinärer Zusammenarbeit hervor. Die Schmerztherapie ergibt sich aus dem Ergebnis des diagnostischen Prozesses und entzieht sich ganz einfachen Empfehlungen. Dem Autor gelang jedoch eine sehr kenntnisreiche Darstellung der Problematik.

Das Update von Jürgen Sartorius über die medikamentöse Schmerztherapie zeigt auf, wie allein über mehr Möglichkeiten der Zubereitung und Zufuhr bekannter Substanzen Fortschritte erreicht werden. Die Basierung der Aussagen auf kontrollierte Studien beinhaltet auch Marketing-Einflüsse, da praktisch nur noch große Konzerne hoch aufwändige und teure Untersuchungen finanzieren können. Aus deutscher Sicht ist interessant, dass die bei uns in Ungnade gefallene AlphaLiponsäure durch neue Studien wieder rehabilitiert wird. Eine wirklich bemerkenswerte Innovation ist der N-TypKalziumkanalblocker Ziconotid mit originärem Wirkansatz und Nebenwirkungsprofil, was zusätzliche Behandlungsmöglichkeiten bei starken, bislang unkontrollierbaren Schmerzzuständen eröffnet. Mit Spannung kann erwartet werden, welche Erfahrungen und eventuell Schwierigkeiten sich bei breiterer Anwendung ergeben.
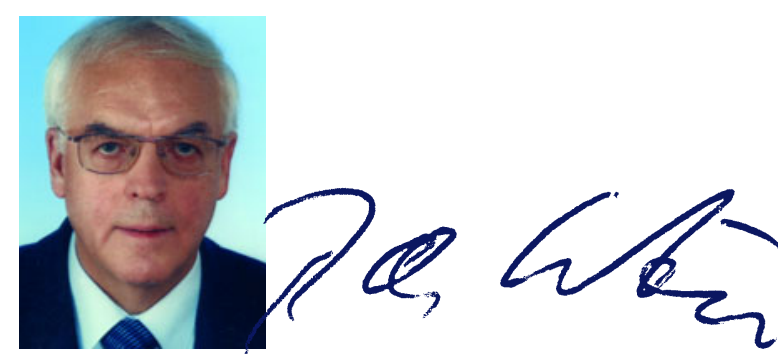

Priv.-Doz. Dr. Roland Wörz, Bad Schönborn 